\title{
Harvesting Beard Hair for Scalp Transplantation
}

\author{
Robert H. True, MD, MPH, FISHRS New York, New York, USA drtrue@hairlossdoctors.com
}

My favorite non-scalp donor area is the beard. Beard hair can be used for a variety of applications to transplant the scalp with good results. Many patients will be able to provide 3,000-5,000 grafts from their beard region. I never try to remove all of the beard hairs, as I think having a thin beard looks more natural than having no beard. With every session, I harvest in an even pattern to maintain a well-distributed but sparser beard.

Harvesting can be challenging. There are typically whorls and significant variation in hair exit angle in the beard (Figure 1). There may be a dozen

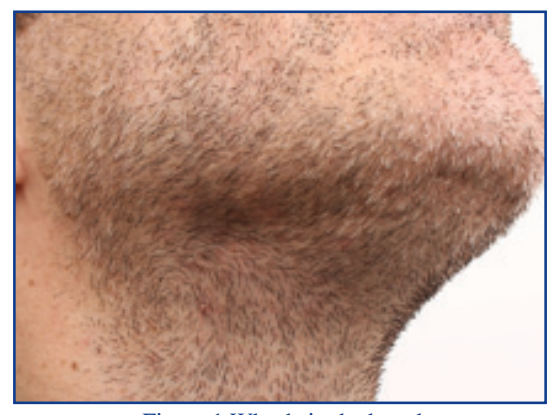

Figure 1 Whorls in the beard or more hair direction changes in a single square centimeter. Beard hairs exit the skin most obliquely along the jawline and submental region. The angle of emergence becomes progressively more acute on the submandibular region and neck as well as the face. It is essential in beard hair harvesting to recognize the blush of anagen hair as it passes through the epidermis (Figure 2). The lowest transection rates are achieved if the punch is centered on this blush rather than the hair shaft as it enters the skin. The technical keys to successful beard hair harvesting are using very sharp

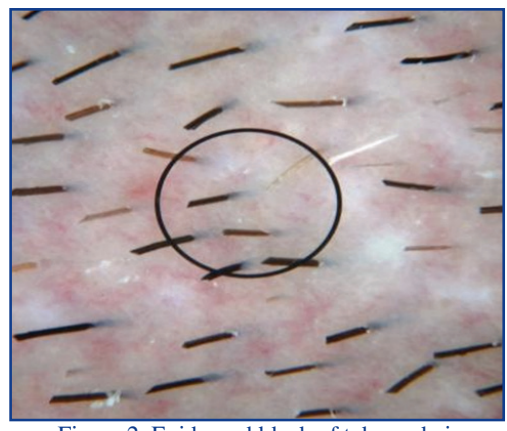
punches and shallow insertions-usually there is no need to go deeper than $2 \mathrm{~mm}$. Because of frequent angle changes, it is helpful to periodically lift and look to see the angle of the hair under the epidermis. Targeting anagen hairs identified by their growth after shaving and the blush produces the best harvest.

The hypermobility of the beard area skin makes extraction difficult. It is critical to provide stretching and tensioning of the skin by having an assistant use two hands to create counter-traction; this is more important than tumescence. Tumescence to large areas is not effective as it disperses too rapidly. I prefer a two forceps extraction method: one to grasp the graft and the other to push the skin adjacent to the extraction site the opposite direction. Applying counter-traction during extraction helps to make extraction easier.

Anesthesia of the entire submandibular region of the beard can be achieved with a ring block (Figure 3). We use $4 \%$ articaine with 1:100,000

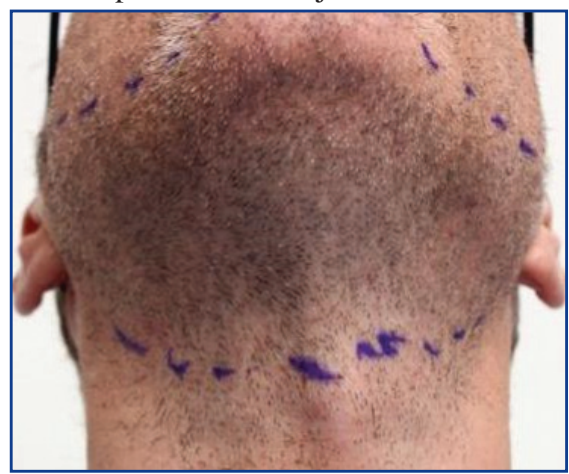

Figure 3. Ring block anesthesia

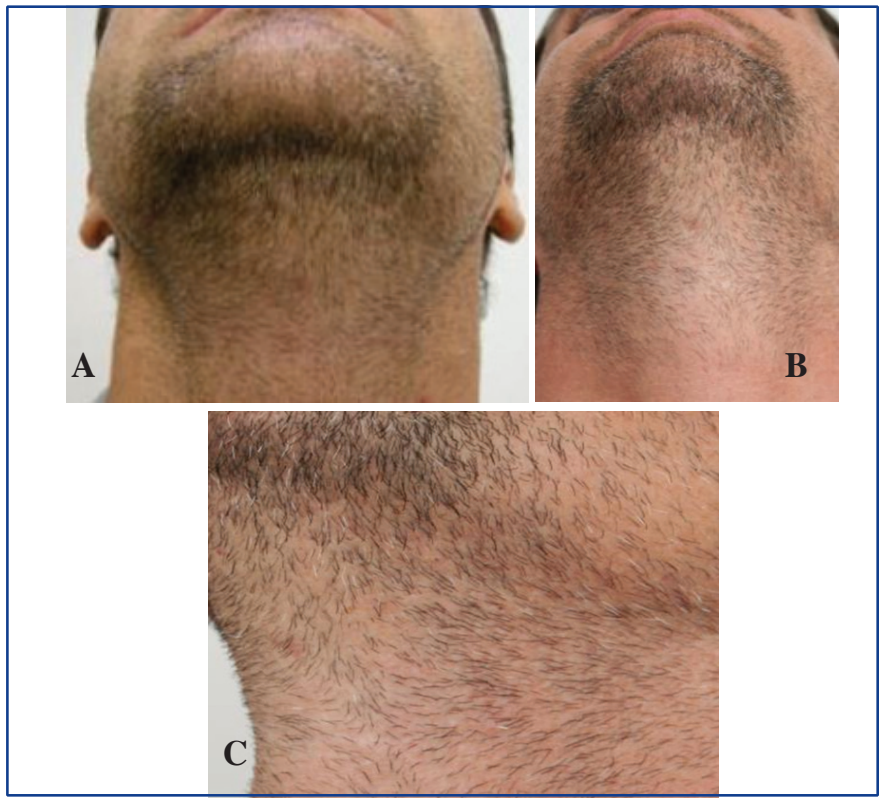

Figure 4. A: Beard area before extraction; $B$ : beard area after extraction of 2,450 grafts; $C$ : close-up of extracted beard.

epinephrine diluted 3:1 with normal saline and then fill the field with $2 \%$ lidocaine with epinephrine 1:100,000 diluted 3:1 saline.

The survival rate of beard hair transplanted to the scalp is uniformly high and I am consistently impressed with how quickly it heals. I also find long-term healing is very good; it is very difficult to discern distinct scars or marks - the beard simply looks thinner as shown in Figure 4 after 2,450 grafts have been harvested. I don't have a lot of experience with patients with dark complexions to know if this remains true for them, but my colleagues in India who perform a lot of beard hair extractions say darker skin also typically heals very well.

I like beard best for donor scars (Figure 5).

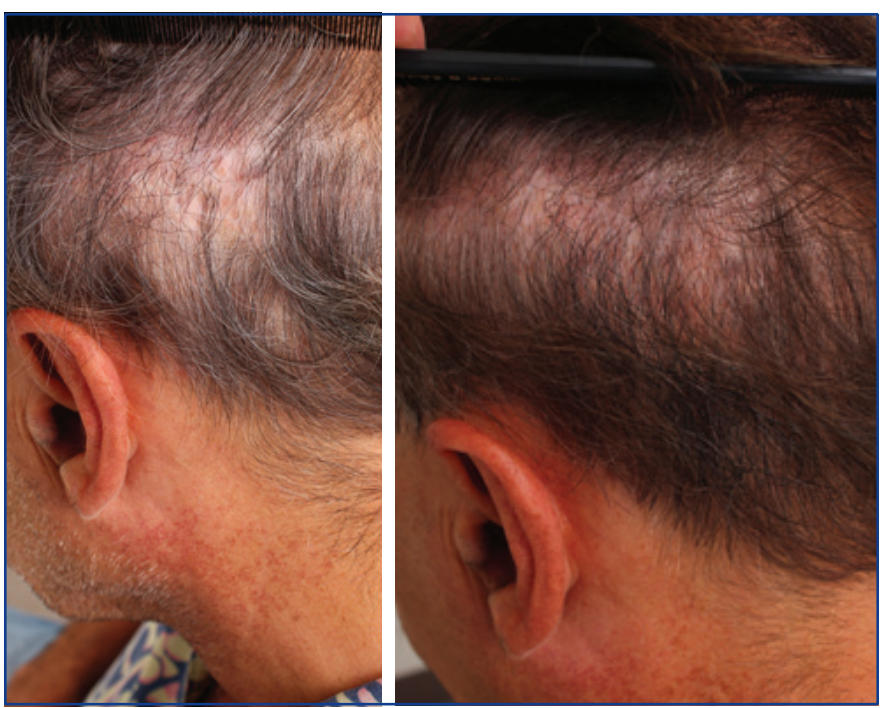

Figure 5. Donor area scar before (left) and after (right) beard hair transplantation.

I think it is a good choice to add density for patients for whom you really want to preserve scalp donor supplies for later (Figure

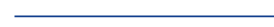




\section{FBeard Chat}

Dr. Robert True to Dr. Kapil Dua: There really isn't literature I can find where beard and moustache density have been studied in a systematic manner.

Dr. Dua: Yes, you are right. I found the one reference in the Kulachi chapter cited in my article, but it doesn't really provide a lot of detail. What I see is mostly surgeons reporting transplanting beards at $25-35 \mathrm{FU} / \mathrm{cm}^{2}$ and moustaches at $35-45 \mathrm{FU} / \mathrm{cm}^{2}$. But what really is the native density and what must we achieve cosmetically? I have measured in around 7 people in my clinic, and it is coming out to be very high, from $45-50 \mathrm{FU} / \mathrm{cm}^{2}$ over the cheek area and $75-80 \mathrm{FU} / \mathrm{cm}^{2}$ over the chin area. Moustache hairs have even higher density of about $85-90 \mathrm{FU} / \mathrm{cm}^{2}$ laterally and $150-160 \mathrm{FU} / \mathrm{cm}^{2}$ in the central area.

Dr. True: These numbers are very high and it is a small sample, but I'm not surprised as I think that Middle Easterners and Southeast Asians have the highest beard densities of all ethnicities.

Dr. Akaki Tsilosani: Concerning the density in beard and moustache areas, according to my experience in Caucasians, density changes between 20-50 FU/cm² (85\% 1 hairs, 10\%, 2 hairs, and less than 5\% 3 hairs). In Asians, density ranges between 20-40 FU/ $\mathrm{cm}^{2}$. I do not have any experience in African cases of beards and moustaches.

Dr. Tony Ruston: In my experience, there are around 30 per $\mathrm{cm}^{2}$ in submandibular and neck areas and 40 in the cheek and moustache areas. I just don't have enough information to contrast ethnic groups.

Dr. Jim Vogel: I would estimate $25-35 \mathrm{FU} / \mathrm{cm}^{2}$. In reality there is no change in density difference that I try to achieve; that is the max that I feel is comfortable within these areas and everyone wants max density.

\section{Beard Hair for Scalp Transplantation from page 155}

6). Usually, beard hair is mixed among scalp hairs, but if scalp and beard hair texture is similar, beard hairs can be exclusively placed in scalp regions (Figure 7). Rarely would I use beard hair for the hairline, but patients with very coarse scalp hair are an exception.

Temporary partial facial paresis can occur in the beard area.

The motor branches of the facial nerve are located in areas
Dr. Jim Harris: I think in someone with a reasonably full beard the density in the buccal area can be 35-50 FU/ $\mathrm{cm}^{2}$. Patients with poor density can be $0-15 \mathrm{FU} / \mathrm{cm}^{2}$. In the submental area, it can be higher like 50-60 FU/ $\mathrm{cm}^{2}$. I think in many patients it drops in the cervical area as compared to the submental region.

I think it's really difficult to categorize by ethnicity. I think patients from the Middle East have had high density, but I've had plenty of Caucasians that have had great density for beard transfer to the scalp, in fact, the beard capacity in some has been greater than 3,000-4,000 grafts.

Dr. Jeffrey Epstein: I am avoidant of talking about density/ $\mathrm{cm}^{2}$ in mathematical terms with all areas including the scalp, just because I find it is one of 3 or 4 major indicators of the appearance of density. In the beard, the most relevant factors are pre-existing presence of hair, color contrast, hair curl, hairs per graft, and density $/ \mathrm{cm}^{2}$.

Dr. Bradley Wolf: I am not a counter at all. I never count densities anywhere. I take what they have and pack them as dense as I can, depending on the number of grafts I have to work with and the space I have to fill. I concentrate on getting them all to grow. I can't help you at all there. I would think it would take a lot of numbers from a lot of patients to standardize densities in different facial areas according to ethnicity. A big undertaking!

Dr. True: What you all are saying is true. It seems that until beard/moustache density studies are done, the best practice is for us to look at the density of native areas of the patient's beard and do our best to cosmetically match that density. Thanks to all for your help in this issue.

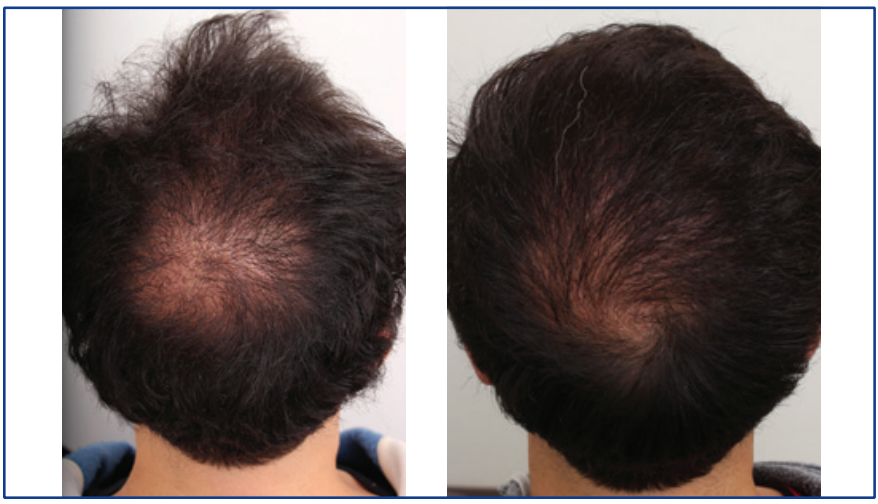

Figure 6. Crown before (left) and after (right) beard hair transplant.

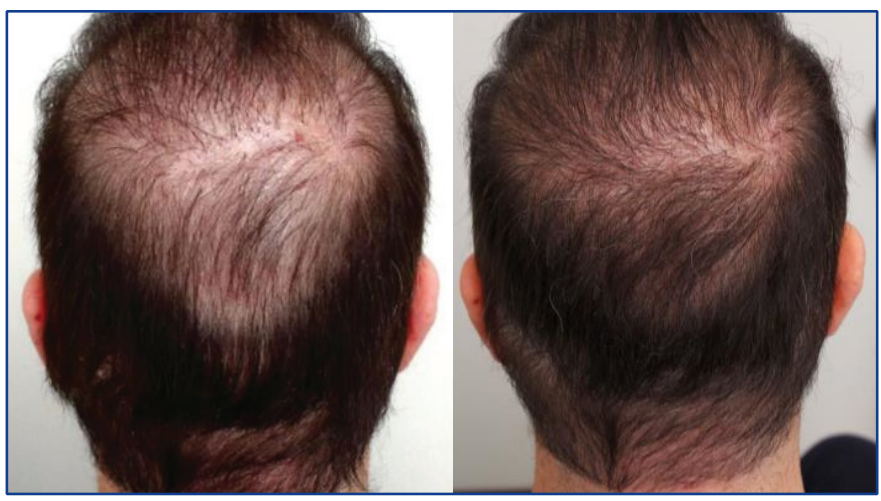

Figure 7. Crown before (left) and after (right) beard hair transplant. 